Design of a low-current shunt-feedback transimpedance amplifier with inherent loop-stability.

M. Mathew, B.L. Hart and K. Hayatleh

School of Engineering, Computing and Mathematics, Oxford Brookes University, Wheatley Campus, Wheatley, Oxford, OX33 1HX, U.K.

maryashok@hotmail.com 


\title{
Design of a low-current shunt-feedback transimpedance amplifier with inherent loop-stability.
}

\begin{abstract}
In this paper we propose a new architecture for enhancing the performance of a transimpedance amplifier (TIA) used for low-currents, and in particular, that used in biosensing. It is usually the first block in biomedical acquisition systems for converting a current in the nanoampere and picoampere range into a proportional voltage, with an amplitude suitable for further processing. There exist two main amplifier topologies for achieving this, current-mode and shunt-feedback mode. This paper introduces a shuntfeedback amplifier that embodies current-mode operation and thereby offers the advantages of both existing schemes. A conventional shunt-feedback amplifier has a number of stages and requires compensation components to achieve stability of the feedback loop. The exemplary circuit described is inherently stable because a high gain is effectively achieved in one stage that has a dominant pole controlling the frequency response. Exhibiting complementary symmetry, the configuration has an input port that is very close to earth potential. This enables the configuration to handle bidirectional input signals such are as met with in electrochemical ampero-metric biosensors. For the $0.35 \mu \mathrm{m}$ process adopted and $\pm 3.3 \mathrm{~V}$ rail supplies, the power dissipation is $330 \mu \mathrm{W}$. With a transimpedance gain of $120 \mathrm{dBohm}$ the incremental input and output resistances are less than 2ohm and the -3dB bandwidth for non-optical input currents is $8.2 \mathrm{MHz}$. The input referred noise current is $3.5 \mathrm{pA} / \sqrt{ } \mathrm{Hz}$.
\end{abstract}

Keywords: Transimpedance amplifier; current to voltage conversion; low input impedance; shunt feedback; biosensing; complementary symmetry 


\section{Introduction.}

A TIA is used in analogue signal processing when a current signal from a sensor is required in voltage form. Sensors are used in a wide range of applications and to set this paper in context, a number of these are listed as references in [1-5]. Various types of biosensors are available, depending on the method of signal acquisition. Among them optical biosensors are the most common. These comprise a bio-recognition sensing element integrated with an optical transducer system [6]. The optical biosensor produces a signal which is proportionate to the concentration of the measured analyte. Then an analogue front-end is required which usually incorporates a transimpedance amplifier (TIA) to convert the current signal to a proportional voltage for further processing in subsequent stages.

Usually, front-end TIAs for optical sensing are designed using a shunt-feedback [7-9], or a current-mode [10] topology. Some of the basic requirements for a TIA design are high gain, good linearity, low-noise and sufficient bandwidth for amplifying a range of biological signals. By achieving a very low input impedance, the TIA can reduce the effects due to the photodiode parasitic capacitance that may limit the bandwidth [11]. In current-mode TIAs, common-gate stages are usually used to achieve low input impedances. In basic shunt-feedback topologies, which use a voltage amplifier with a feedback network, the input impedance is decreased by the open-loop gain of the voltage amplifier. Current-mode topologies are stable and provide wide bandwidth. However, they have a higher input-referred noise current. The shunt-feedback topologies have comparatively lower input-referred noise current. In this paper, incorporating the benefits of both the topologies, we propose a topology which gives a better performance than the current-mode and conventional shunt-feedback topologies. 
The proposed TIA is designed with a single-stage high transimpedance currentto-voltage amplifier with shunt-feedback. The design exhibits complementary symmetry about earth potential and therefore benefits from having an input that is very close to earth. It also has low input and output impedances (in the low-ohmic region), high transimpedance gain from a single stage and low input-referred noise current. Furthermore, in this design no compensation components are necessary to ensure frequency stability of the feedback loop as it offers inherent stability, for a wide range of feedback resistors.

\section{Circuit architecture and analysis}

The first part of this section considers notable points in the architecture, the second part is a circuit analysis from first principles.

The proposed circuit shown in Fig.1 comprises five interconnected vertical branches (B1-B5). A complimentary grounded-gate input stage forms the input port X to the shunt-feedback amplifier and provides a low incremental input resistance. The input current $i_{i}$, at $\mathrm{X}$, to the amplifier is $\left(i_{s}-i_{f}\right)$ where $i_{s}, i_{f}$ are respectively, the signal current being monitored and the feedback current supplied for the amplifier output voltage $v_{o}$ at Y. It is shown below that, to a high degree of predictability, $v_{o}=-R_{F} i_{s}$.

The DC currents $I_{1}, I_{2}$ in branch B1 determine the bias current, $I_{B}$, in each of the following stages. Then if $\mathrm{I}_{1}=\mathrm{I}_{2}$ and $\mathrm{M}_{3}, \mathrm{M}_{10}$ and $\mathrm{M}_{4}, \mathrm{M}_{11}$ are well-matched pairs, the bias current $I_{B}$ in branch $B_{2}$ is equal to $I_{2}$ and $X$ is at, or very near, earth potential in the absence of a signal current. However, this is only true if the gate-drain voltage of $\mathrm{M}_{10}$ is the same as that of $\mathrm{M}_{3}$ and that of $\mathrm{M}_{11}$ the same as that of $\mathrm{M}_{4}$. To help ensure this, both $\mathrm{M}_{10}$ and $\mathrm{M}_{11}$ are doubly cascoded. 


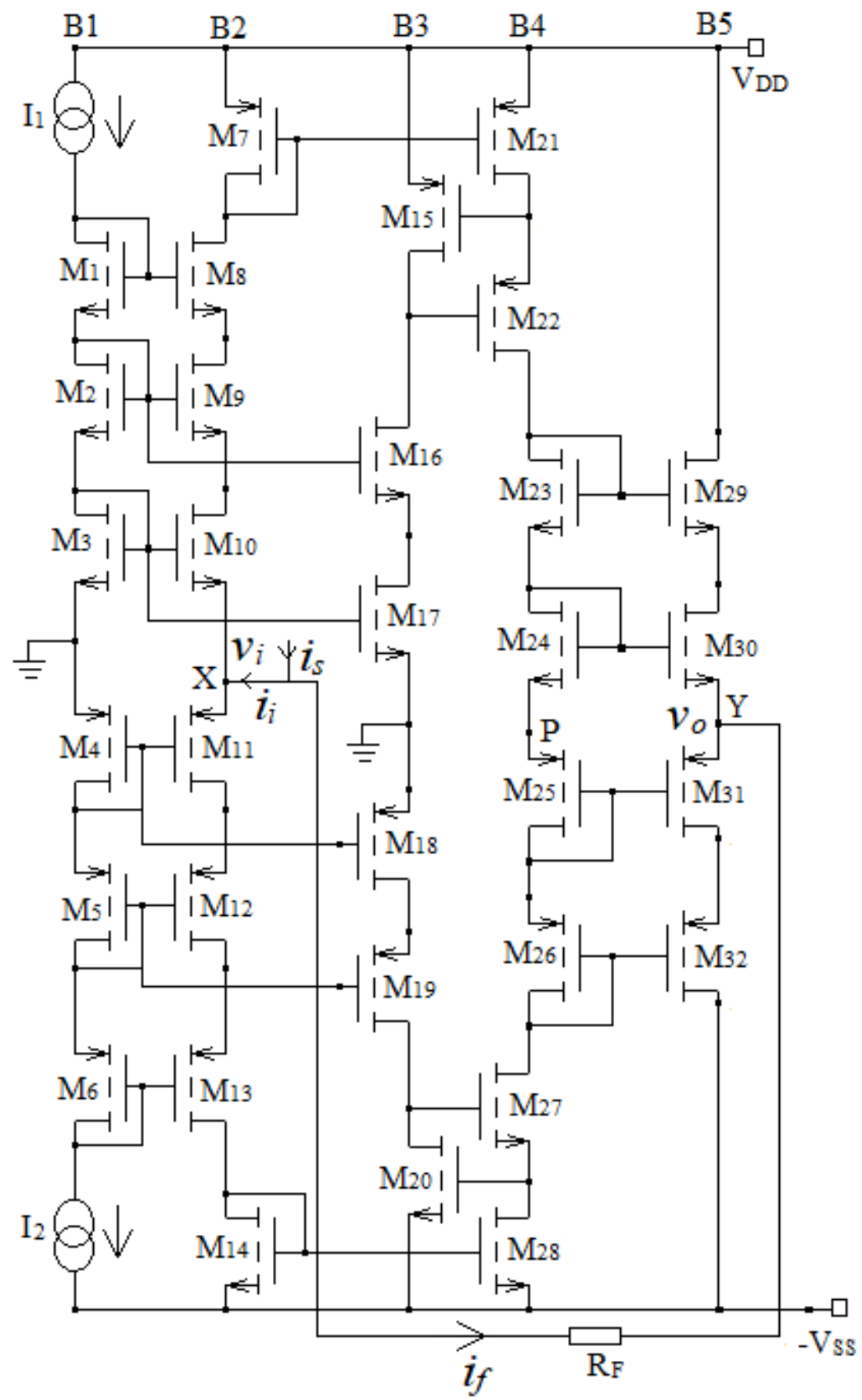

Fig.1. Proposed new Transimpedance Amplifier Circuit.

Signal current, $i_{s}$, causes a change in the currents of $\mathrm{M}_{7}$ and $\mathrm{M}_{14}$, which are the input mosfets to the complementary regulated cascodes (RGCs) formed by $\mathrm{M}_{15}, \mathrm{M}_{21}$, $\mathrm{M}_{22}$ and $\mathrm{M}_{20}, \mathrm{M}_{28}, \mathrm{M}_{27}$. Amplifying transistors $\mathrm{M}_{15}, \mathrm{M}_{20}$ are supplied with drain-current loads to maximize their voltage gains and thus maximize the output resistances at the 
drains of $\mathrm{M}_{22}$ and $\mathrm{M}_{27}$. The voltage change, at point $\mathrm{P}$, due to $i_{s}$ appears at $\mathrm{X}$ via the complementary source-follower formed by $\mathrm{M}_{24}, \mathrm{M}_{30}$ and $\mathrm{M}_{25}, \mathrm{M}_{31}$. These pairs are cascoded to ensure that the DC voltage at $\mathrm{X}$ is close to that at $\mathrm{P}$.

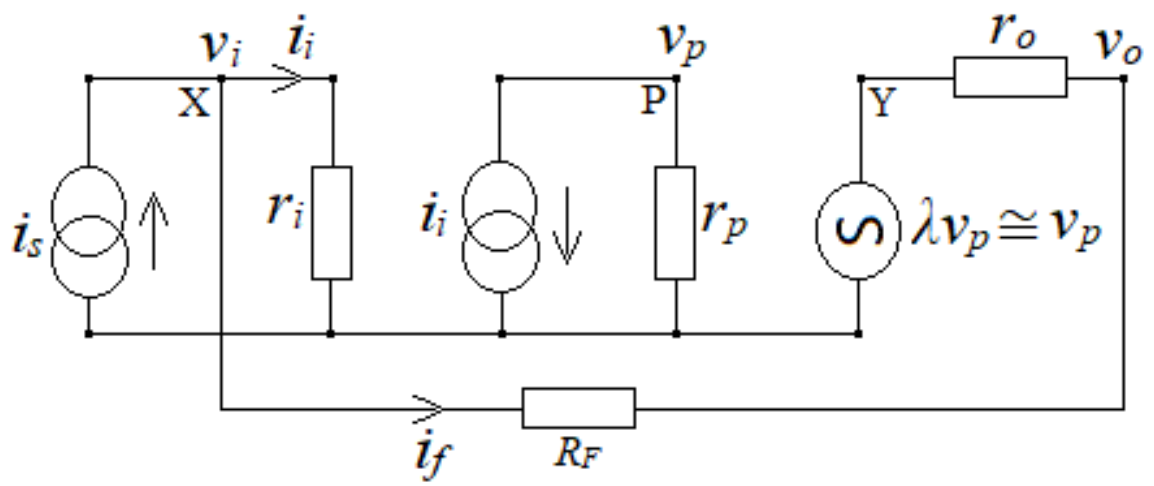

Fig.2 Small-signal low-frequency equivalent circuit of Fig.1

The points $\mathrm{X}, \mathrm{P}$, and $\mathrm{Y}$ of Fig.1 are shown on the corresponding small-signal low-frequency equivalent circuit of Fig.2, which serves for a circuit analysis from first principles: $r_{i}$ and $r_{o}$ are respectively, the incremental input resistance at $\mathrm{X}$ and the output resistance at $\mathrm{Y}$ in the absence of feedback; $r_{p}$ is the incremental resistance looking in at point $\mathrm{P}$; and, $\lambda$ is the voltage gain of the source-follower.

There is no loss in principle, and no significant loss in accuracy in a first-order analysis, in making three simplifying assumptions. First, the current-mirrors have a current transfer coefficient of precisely unity, and second that all the N-channel mosfets have a common value, $g_{m n}$ of transconductance and $r_{o n}$ for output resistance. The corresponding common values for P-channel mosfets are $g_{m p}, r_{o p}$. The third assumption is that $\lambda=1$.

$$
\text { At } \mathrm{X}, r_{i}=\frac{1}{\left(g_{m n}+g_{m p}\right)}
$$

Similarly, at Y, $r_{o}=\frac{1}{\left(g_{m n}+g_{m p}\right)}$

When $i_{s}$, is applied, flowing in the direction shown, the input voltage $v_{i}$ is given by 
$v_{i}=\frac{i_{i}}{\left(g_{m n}+g_{m p}\right)}$

The resultant current change in $\mathrm{M}_{11}$ is $g_{m p} v_{i}=\frac{g_{m p} i_{i}}{\left(g_{m n}+g_{m p}\right)}$ and that in $\mathrm{M}_{10}$ is

$-g_{m n} v_{i}=\frac{-g_{m n} i_{i}}{\left(g_{m n}+g_{m p}\right)}$. Following subsequent current-mirror action, these combine to form a current $i_{i}$, that produces a voltage change $v_{p}=-i_{i} r_{p}$, at $\mathrm{P}$.

From [12], we can write,

$r_{p=}\left[r_{o p}\left(g_{m p} r_{o p}\right)^{2} \|\left[r_{o n}\left(g_{m n} r_{o n}\right)^{2}\right]\right]$

The open-circuit voltage of the source-follower is $v_{p}$ assuming $\lambda=1$.

By inspection, $v_{i}-v_{p}=i_{f}\left(R_{F}+r_{o}\right)$,

Or, $i_{i} r_{i}-\left(-i_{i} r_{p}\right)=i_{f}\left(R_{F}+r_{o}\right)$.

Substituting $i_{i}=i_{s}-i_{f}$ into equation (5) gives, after routine algebraic manipulation,

$i_{f}=\frac{i_{s}}{\left\{1+\frac{\left(R_{F}+r_{o}\right)}{\left(r_{p}+r_{i}\right)}\right\}}$

And,

$i_{i}=\frac{i_{s}}{\left\{1+\frac{\left(r_{p}+r_{i}\right)}{\left(R_{F}+r_{0}\right)}\right\}}$

Furthermore, $v_{o}=v_{i}-i_{f} R_{F}$

Thus from (6), (7),

$v_{0}=\frac{i_{S} r_{i}}{\left\{1+\frac{\left(r_{p}+r_{i}\right)}{R_{F}+r_{o}}\right\}}-\frac{i_{S} R_{F}}{\left\{1+\frac{\left(R_{F}+r_{o}\right)}{\left(r_{p}+r_{i}\right)}\right\}}$

By design, $r_{p} \gg R_{F} \gg r_{i}, r_{o}$, so the first term in equation (9) can be ignored compared with the second and we can write,

$v_{0} \cong \frac{-i_{s} R_{F}}{\left\{1+\frac{R_{F}}{r_{p}}\right\}}$

Or, in practice for DC and low frequency operation $v_{o} \cong-i_{s} R_{F}$, 
The quantity $\frac{\left(r_{p}+r_{i}\right)}{\left(R_{F}+r_{o}\right)}$ is identified as the loop gain taking into account the input and output resistances. A straightforward classical feedback approach ignores these. The input and output resistances with feedback are those without feedback reduced by the magnitude of the loop gain and are given by,

$$
r_{i f}=r_{o f} \cong \frac{r_{i} R_{F}}{r_{p}}
$$

Consider now the frequency dependence of the trans-admittance. A cursory examination of Fig.1 using the method of 'Zero Value Time Constant Analysis' [13] suggests that a dominant time-constant might be $r_{p} C_{o}$, $C_{o}$ being the capacitance at $\mathrm{P}$, because all other nodal capacitances are of a similar order of magnitude but $r_{p}$ is by far the greatest associated resistance. In that case, in terms of the complex frequency variable s,

$Z_{p}(s)=\frac{r_{p}}{\left(1+s C_{o} r_{p}\right)}$

Substituting this in place of $r_{p}$ in equation (10) with the proviso $r_{p} \gg R_{F}$ finally gives,

$Z_{T}(s)=\frac{v_{o}}{i_{s}}(s)=\frac{-R_{F}}{\left(1+s C_{o} R_{F}\right)}$

The corresponding cut-off frequency, $f_{c}$ is inversely related to $R_{F}$ and is given by,

$f_{c}=\frac{1}{\left(2 \pi C_{o} R_{F}\right)}$

\section{Results}

In a Cadence Simulation of Fig.1 the component choice was as follows: for all the Mosfets $\mathrm{L}=0.35 \mu \mathrm{m} ; \mathrm{M}_{22}-\mathrm{M}_{27}$ and $\mathrm{M}_{29}-\mathrm{M}_{32}$ had $\mathrm{W}=5 \mu \mathrm{m}$; for the rest $\mathrm{W}=10 \mu \mathrm{m}$. Operating conditions were $V_{D D}=-V_{S S}=3.3 \mathrm{~V}$ and $\mathrm{I}_{1}=\mathrm{I}_{2}=10 \mu \mathrm{A}$. 


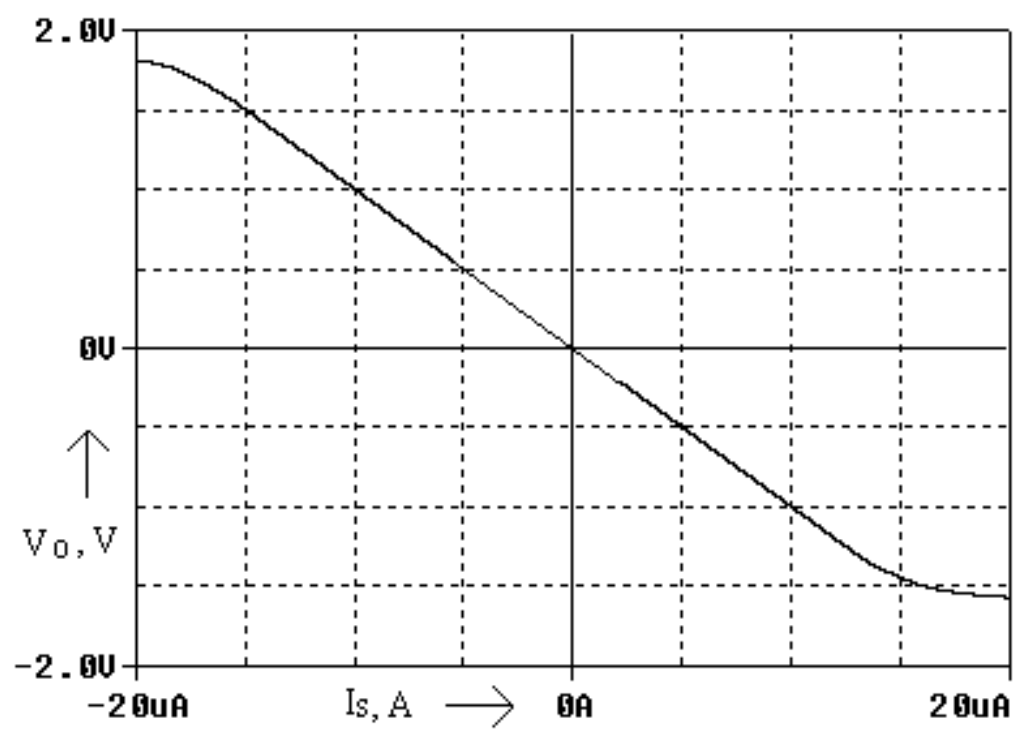

Fig.3a DC transfer characteristics of the proposed TIA for $\mathrm{R}_{\mathrm{F}}=100 \mathrm{k} \Omega$

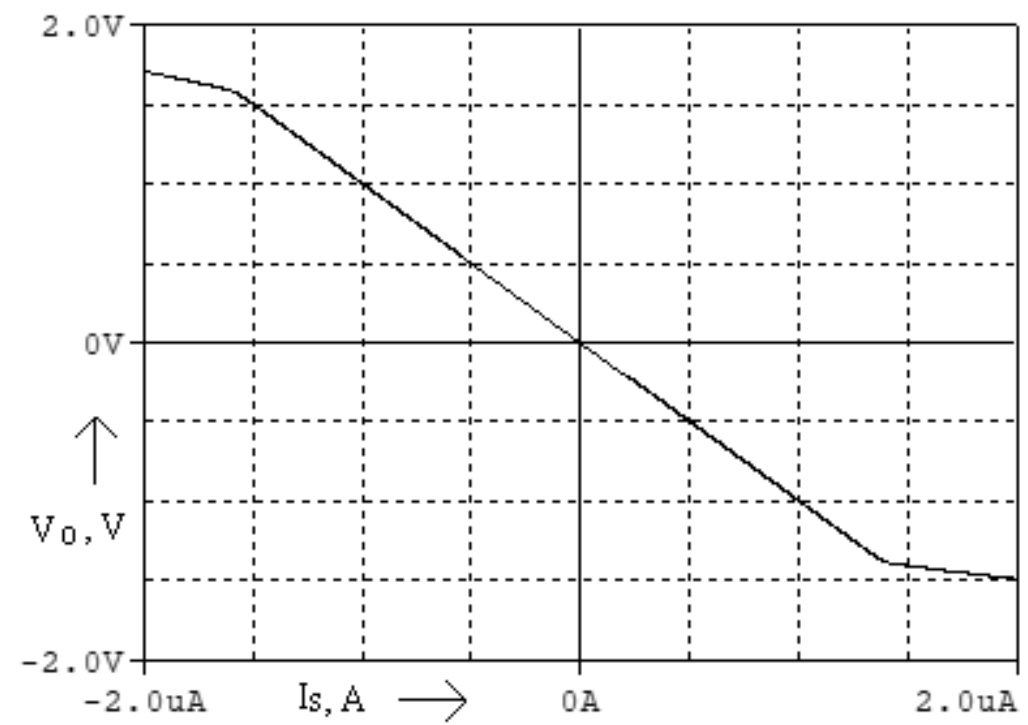

Fig.3b DC transfer characteristics of the proposed TIA for $\mathrm{R}_{\mathrm{F}}=1 \mathrm{M} \Omega$.

Figs 3a,3b show, respectively, the DC transfer characteristics for $\mathrm{R}_{\mathrm{F}}=100 \mathrm{k} \Omega$ and $\mathrm{R}_{\mathrm{F}}=1 \mathrm{M} \Omega$. These resistances are chosen to give a guaranteed output voltage magnitude of $1 \mathrm{~V}$ for $I_{S}=10 \mu \mathrm{A}$ and $I_{S}=1 \mu \mathrm{A}$ respectively. The graphs appear to be linear and pass through the origin (however, a separate check showed a $330 \mu \mathrm{V}$ offset from earth). For Vo $>1.5 \mathrm{~V}$ in Fig.3a there is non-linearity because of the onset of triode behaviour in the 
P- channel RGC formed by $\mathrm{M}_{15}, \mathrm{M}_{21}$ and $\mathrm{M}_{22}$. There is also non-linearity for $\mathrm{V}<-$ $1.25 \mathrm{~V}$, because of the onset of triode behaviour in the RGC formed from $\mathrm{M}_{20}, \mathrm{M}_{27}, \mathrm{M}_{28}$.

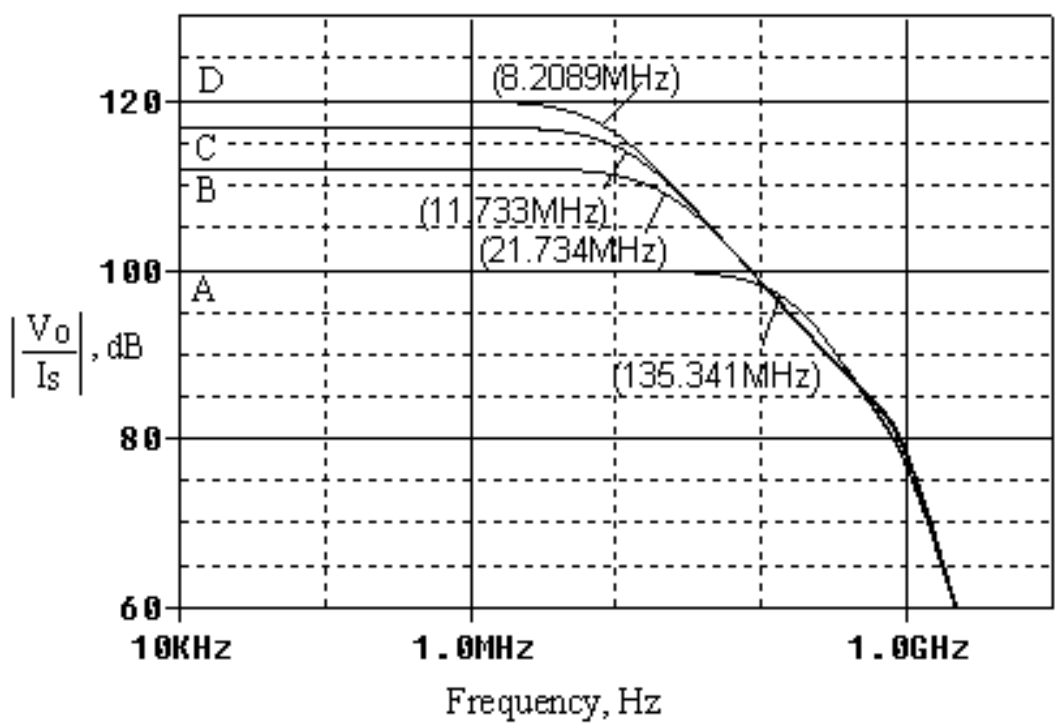

Fig.4a Frequency response plots A, B, C, D refer respectively to $R_{F}=100 \mathrm{k} \Omega$, $400 \mathrm{k} \Omega, 700 \mathrm{k} \Omega$ and $1 \mathrm{M} \Omega$.

In the frequency response plots of Fig.4a, for non-optical current inputs the curves labelled A, B, C, D refer, here and henceforth, respectively, to $R_{F}=100 \mathrm{k} \Omega$, $400 \mathrm{k} \Omega, 700 \mathrm{k} \Omega, 1 \mathrm{M} \Omega$. The $-3 \mathrm{~dB}$ cut off-frequency, $f_{c}$, is shown by each curve. With $\mathrm{R}_{\mathrm{F}}$ $=1 \mathrm{M} \Omega(120 \mathrm{~dB} \Omega$ for a dB reference level of $1 \Omega), f_{c}=8.209 \mathrm{MHz}$ : with $\mathrm{R}_{\mathrm{F}}=700 \mathrm{k} \Omega$, $f_{c}=11.73 \mathrm{MHz}$ compared with a value of 11.72 predicted from equation (15) for the same value of $C_{o}$. Similarly, for $\mathrm{R}_{\mathrm{F}}=400 \mathrm{k} \Omega, f_{c}=21.734$ compared with a predicted value of $20.52 \mathrm{MHz}$, which is only $4.4 \%$ lower. However, equation (15) does not apply for $100 \mathrm{k} \Omega$ itself. The break in the trade-off between gain and frequency response is because with high loop-gains, the frequency response is not controlled by a single dominant pole [14]. Other poles and a zero make their presence felt.

Stability is assured for $\mathrm{R}_{\mathrm{F}}=100 \mathrm{k} \Omega$ for which the phase margin is $68^{\circ}$, and is also for operation below $100 \mathrm{k} \Omega$. Thus, for $\mathrm{R}_{\mathrm{F}}=50 \mathrm{k} \Omega$, the phase margin is $51^{\circ}$. 
However, operation below $\mathrm{R}_{\mathrm{F}}=100 \mathrm{k} \Omega$ is not recommended with input currents from photodiodes, as will be evident from the undesirable peak in the frequency response characteristics displayed below. The peak increases as $\mathrm{R}_{\mathrm{F}}$ gets progressively smaller.

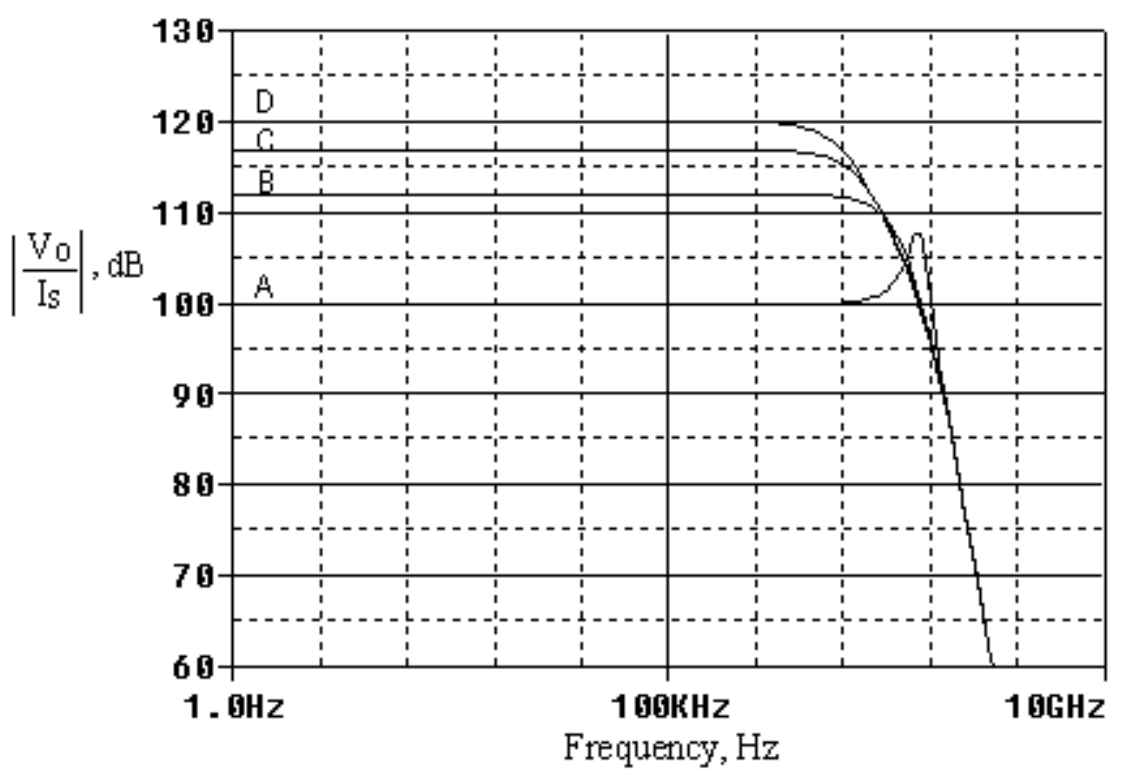

Fig.4b. Frequency response plots with photodiode junction capacitance of $0.5 \mathrm{pF}$ at the input and load capacitance of $0.1 \mathrm{pF}$.

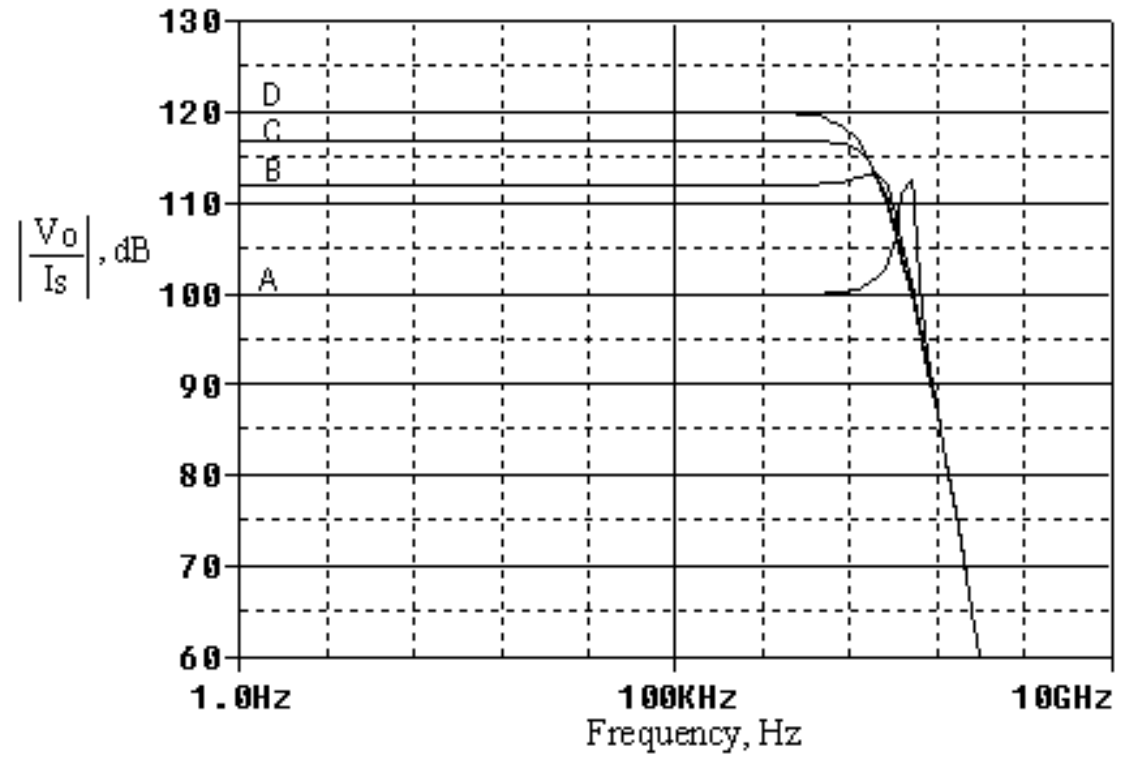

Fig.4c. Frequency response plots with photodiode junction capacitance of $2 \mathrm{pF}$ at the input and load capacitance of $0.1 \mathrm{pF}$. 
In Fig.4b, 4c, plots A, B, C, D refer respectively to resistance values mentioned earlier. The capacitance used refer to two types of photodiode junction capacitance. The $0.1 \mathrm{pF}$ refers to the likely load of a mosfet load in a succeeding stage.

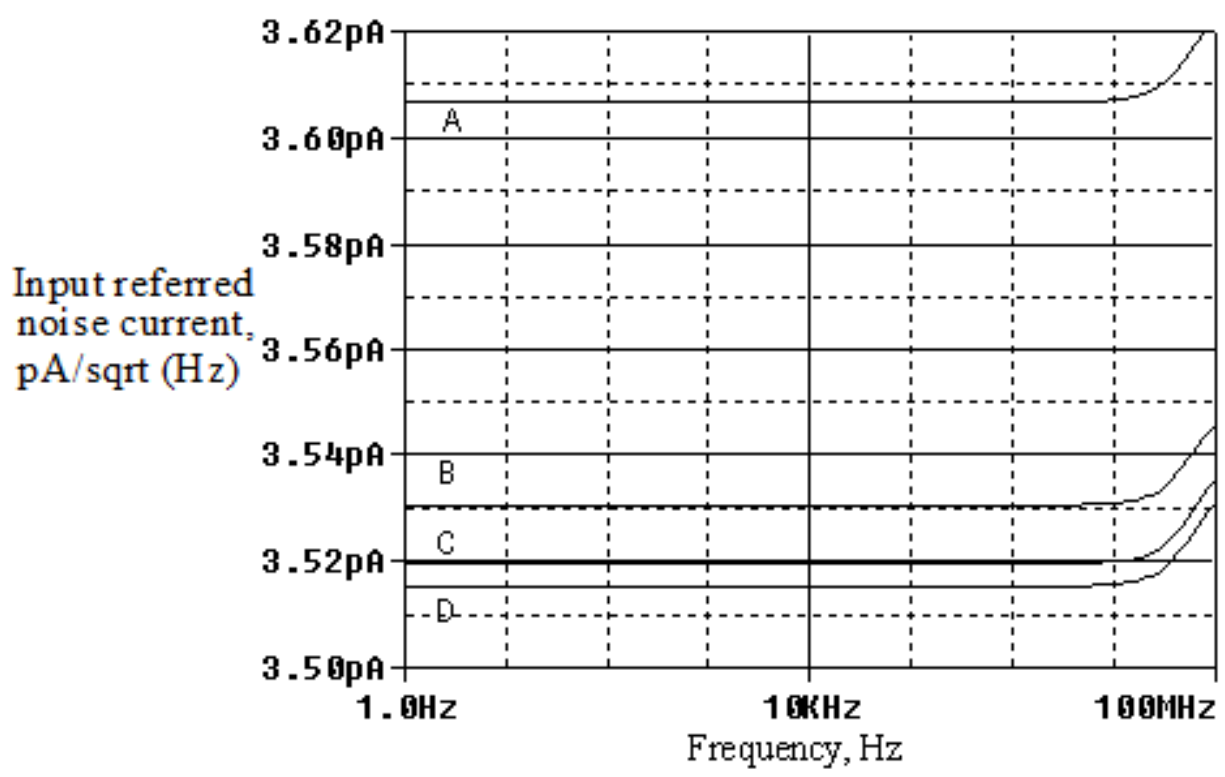

Fig.5 Input referred noise current $(\mathrm{A} / \sqrt{ } \mathrm{Hz})$ of TIA with photodiode junction capacitance $2 \mathrm{pF}$ and load capacitance $0.1 \mathrm{pF}$. The plots $\mathrm{A}, \mathrm{B}, \mathrm{C}$, D refer respectively to $\mathrm{R}_{\mathrm{F}}=100 \mathrm{k} \Omega$, $400 \mathrm{k} \Omega, 700 \mathrm{k} \Omega$, and $1 \mathrm{M} \Omega$.

For frequencies below 10MHz, Fig.5 shows the input referred noise current which is the r.m.s output noise voltage divided by the TIA gain. In the proposed configuration the gain is high without feedback instability because the frequency response is dominated by a single pole. That is why the input referred noise current $(3.5 \mathrm{pA} / \sqrt{ } \mathrm{Hz})$ is significantly lower than that in the circuits described in [15].

Investigation showed that most of the noise occurred in the current mirrors [14], $\mathrm{M}_{7}, \mathrm{M}_{21}$ and $\mathrm{M}_{14}, \mathrm{M}_{28}$ of Fig.1. It can be reduced by operating with a lower bias current, e.g., with $I_{B}=5 \mu \mathrm{A}$, the input referred noise current fell to $2.56 \mathrm{pA} / \sqrt{ } \mathrm{Hz}$. However, operating with a lower $\mathrm{I}_{\mathrm{B}}$ means a lower range of input currents that can be measured, without using a much higher value of $\mathrm{R}_{\mathrm{F}}$, and a higher input resistance. 


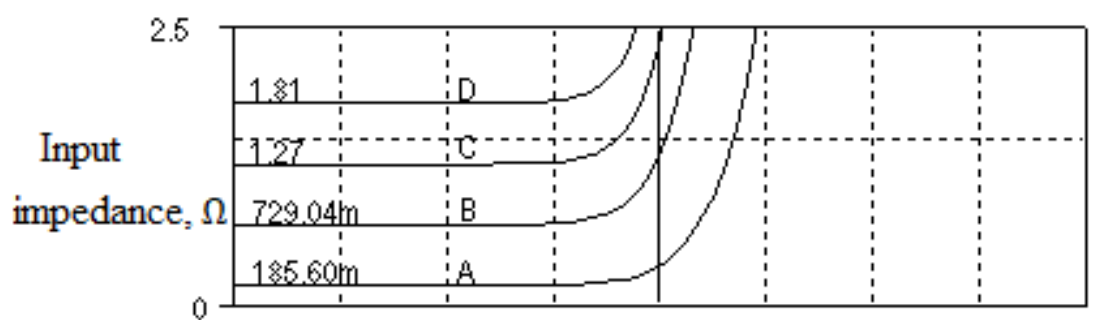

(a)

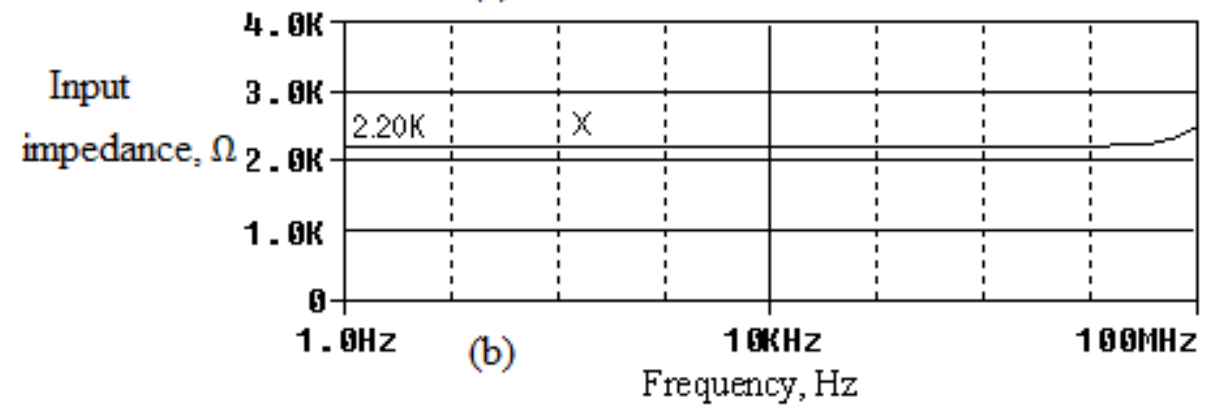

Fig.6 In (a) Plots A, B, C, D are input impedance plots with feedback resistors as mentioned earlier and in (b) plot X refer to input impedance without feedback resistor.

In Fig.6 the dramatic reduction in input impedance for each feedback resistance to less than $2 \Omega$ for curves A, B, C, D from its non-feedback value of more than $2 \mathrm{k} \Omega$ in curve $\mathrm{X}$ is evidence of the very high loop-gain.

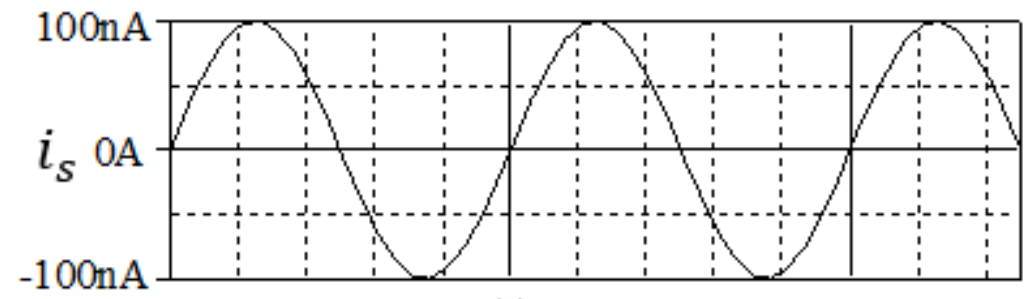

(a)

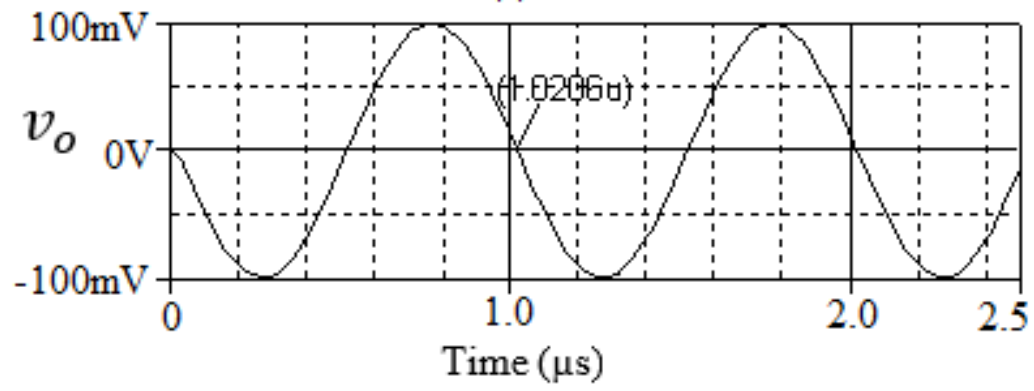

(b)

Fig.7 (a) Input signal, $i_{s}$ (b) Output signal, $v_{o}$ with $\mathrm{R}_{\mathrm{F}}=1 \mathrm{M} \Omega$.

As an illustration of the performance of the proposed TIA with a bidirectional current input, Fig.7 shows the output for a $1 \mathrm{MHz}$, sinusoidal input with 100nA peak. In 
this particular case there is an output delay of approximately 20nS. Table 1 shows the performance of the proposed circuit compared with that mentioned in [15].

Table 1 Performance comparison

\begin{tabular}{|l|l|l|l|l|}
\hline Circuit & \multicolumn{2}{|l|}{ Proposed circuit } & $\begin{array}{l}\text { Shunt-feedback } \\
{[15]}\end{array}$ & $\begin{array}{l}\text { Current-mode } \\
{[15]}\end{array}$ \\
\hline Transimpedance & $120 \mathrm{~dB} \Omega$ & $100 \mathrm{~dB} \Omega$ & $112 \mathrm{~dB} \Omega$ & $83 \mathrm{~dB} \Omega$ \\
\hline Bandwidth & $8.2 \mathrm{MHz}$ & $135 \mathrm{MHz}$ & $2 \mathrm{MHz}$ & $115 \mathrm{MHz}$ \\
\hline Input impedance & $<2 \Omega$ & $<0.2 \Omega$ & - & $145 \Omega$ \\
\hline Input-referred noise & $* 3.5 \mathrm{pA} / \sqrt{\mathrm{Hz}}$ & $* 3.5 \mathrm{pA} / \sqrt{ } \mathrm{Hz}$ & $185 \mathrm{pA} / \sqrt{\mathrm{Hz}}$ & $53 \mathrm{nA} / \sqrt{\mathrm{Hz}}$ \\
\hline Process Technology & $0.35 \mu \mathrm{m}$ & $0.35 \mu \mathrm{m}$ & $0.18 \mu \mathrm{m}$ & $0.18 \mu \mathrm{m}$ \\
\hline Power Dissipation & $330 \mu \mathrm{W}$ & $330 \mu \mathrm{W}$ & $0.5 \mathrm{~mW}$ & $28.6 \mathrm{~mW}$ \\
\hline
\end{tabular}

\section{Conclusions}

A proposed low-current shunt-feedback transimpedance amplifier can be designed so that it does not require compensation components to ensure stability of the feedback loop because it uses only one high gain stage instead of the three lower gain stages of a conventional transimpedance amplifier. Cadence simulation results show very low input and output resistances, a high transimpedance gain and bandwidth that make it suitable for biosensing measurements.

\section{Acknowledgements}

The authors would like to thank the reviewers for their constructive comments. 
References

[1]. Zheng, H., Ma, R., Liu, M., Zhu, Z. (2018). High Sensitivity and Wide Dynamic Range Analog Front-End Circuits for Pulsed TOF 4-D Imaging LADAR Receiver. IEEE Sensors Journal, 18(8),3114-3124.

[2]. Zheng, H., Ma, R., Liu, M., Zhu, Z. (2018). A Linear Dynamic Range Receiver with Timing Discrimination for Pulsed TOF Imaging LADAR Application. IEEE Trans. on Instrumentation \& Measurement, 67(11), 2684-269.

[3]. Zheng, H., Ma, R., Zhu, Z. (2017). Design of Linear Dynamic Range and High Sensitivity Matrix Quadrant APDs ROIC for Position Sensitive Detector Application. Microelectronics Journal,63, 49-57.

[4]. Z Zheng, H., Ma, R., Zhu, Z. (2017). A Linear and Wide Dynamic Range Transimpedance Amplifier with Adaptive Gain Control Technique. Analog Integrated Circuits and Signal Processing, 90(1),217-226.

[5]. Ma, R., Zheng, H., Zhu, Z. (2017) A high sensitive 66 dB linear dynamic range receiver for 3-D laser radar. Journal of Semiconductors,38(8),81-86.

[6]. Damborsky, P., Svitel, J., Katrlík, J. (2016). Essays in Biochemistry, 60(1) 91100; DOI: 10.1042/EBC20150010

[7]. Sackinger, E. (2010). The transimpedance limit, IEEE Trans. Circuits Syst. I Reg. Papers, 57(8), 1848-1856.

[8]. Sedra A., \& Smith K. (2011). Chapter 9, International Student Edition, Microelectronic Circuits. (pp. 814 - 822). New York: Oxford University Press.

[9]. Ibrahim M., Levine P., Hirano, A. (2014). CMOS transimpedance amplifier for biosensor signals. IEEE International Symposium on Circuits and Systems, Melbourne VIC, Australia, June 2014, pp. 25-28., doi: 10.1109/ISCAS.2014.6865056

[10]. Park S. M., Toumazou C. (1997). Gigahertz low noise CMOS transimpedance amplifier. Proceedings of IEEE International Symposium on Circuits and Systems, 1, 209-212.

[11]. Ma, R., Liu, M., Zheng, H., Zhu, Z. (2018). A 77-dB Dynamic Range LowPower Variable-Gain Transimpedance Amplifier for Linear LADAR. IEEE Transactions on Circuits and Systems II: Express Briefs. 65(2), 171-175.

[12]. Allen P.E., Holberg D. R. (2002). Chapter 4, Second Edition, CMOS Analog Circuit Design (pp. 141). New York: Oxford University Press. 
[13]. Gray P.R., Hurst P. J., Lewis S. H., Meyer R. G. (2001). Chapter 7, Fifth Edition, Analysis and Design of Analog Integrated Circuits (pp. 518-527). U.S.A: John Wiley and Sons.

[14]. Sackinger, E., Guggenbuhl, W. (1990). A high swing, high impedance MOS cascode circuit. IEEE Journal of Solid-State Circuits, 25(1), 289-298.

[15]. Trabelsi, A., Boukadoum, M. (2012). A Comparative Overview of Two Transimpedance Amplifiers for Biosensing Applications. IEEE International Symposium on Circuits and Systems, 2227-2230. 